\title{
Summary and Prospect of Treating Ulcerative Colitis by Warming the Kidney and Strengthening the Spleen
}

\author{
Liyan Liu' ${ }^{*}$, Xiaoquan $\mathrm{Du}^{2}$ \\ 712000, Shaanxi Province, China \\ *Corresponding author: Liyan Liu, yan15709100977@163.com
}

${ }^{1}$ Shaanxi University of Traditional Chinese Medicine, Xianyang 712000, Shaanxi Province, China

${ }^{2}$ The $2^{\text {nd }}$ Digestive Department, Affiliated Hospital of Shaanxi University of Traditional Chinese Medicine, Xianyang

\begin{abstract}
The method of warming the kidney and strengthening the spleen is widely used in the treatment of ulcerative colitis (UC) with significant curative effect. This paper reviews the relevant literature in recent years.
\end{abstract}

Keywords: Ulcerative colitis; Method of warming kidney and strengthening spleen

Publication date: July 2021; Online publication: July 31, 2021

\section{Introduction}

The main clinical manifestations of UC are abdominal pain, diarrhea, mucus purulent and bloody stool, which often occur repeatedly and are difficult to heal. Its etiology and mechanism are not clear, and there is no specific treatment at present. Traditional Chinese medicine (TCM) has obvious advantages in relieving symptoms, preventing recurrence and reducing complications. The recent research progress of warming the kidney and strengthening the spleen in the treatment of UC is summarized as follows.

\section{Warming the kidney and strengthening the spleen is the main treatment for UC}

\subsection{The internal treatment method}

Warming the kidney and strengthening the spleen is widely used in clinical practice. Professor Bian Songjing ${ }^{[1]}$ thinks that the remission stage of UC is mainly due to Yang deficiency of the spleen and kidney, which is combined with local damp heat of the large intestine. The treatment principle is warming the kidney and strengthening the spleen, clearing the Intestine and leading stagnation, and the drugs are used together with the operation to achieve the effect of warming the kidney and strengthening the spleen; Professor Liu Yanjing ${ }^{[2]}$ thinks that spleen qi deficiency is the root of UC, and kidney yang deficiency is the inevitable outcome in the development of UC. Dampness evil runs through the whole process of UC. The main treatment is warming kidney, strengthening spleen and removing dampness. Sishen pill and Linggui Zhugan decoction are used to treat UC.

In recent years, many doctors have further confirmed that the method of warming the kidney and strengthening the spleen is effective in the treatment of UC. Xing Wei ${ }^{[3]}$ and others took mesalazine enteric coated tablets combined with Sishen pill and Linggui Zhugan Decoction to treat UC. After 12 weeks of treatment, the results showed that the total effective rate of the treatment group was significantly higher than that of the control group. He Qinghua ${ }^{[4]}$ and others selected 61 cases of UC with spleen kidney yang deficiency syndrome. The treatment group was treated with Renren yangzang decoction combined with Nuangan Decoction on the basis of the treatment of the control group. After 4 weeks of treatment, it was 
finally proved that Wenbu PI Shen method could significantly improve the curative effect of TCM syndrome in UC. Lu maoyong et al ${ }^{[5]}$ randomly divided 72 patients into the experimental group and the control group. The control group was treated with mesalazine, and the experimental group was treated with self-made Sishen pill (Buguzhi, simmered nutmeg, tufuling each 15g, prepared aconite, chuanxiong, Wumei, Schisandra, fried Fructus aurantii, angelica each 10g, Huangqi, Baizhu each 20g, Evodia rutaecarpa 5g, Huanglian 6g), After two months of treatment, the results showed that the improvement of TCM symptoms in the experimental group was significantly better than that in the control group. Zhang Luding ${ }^{[6]}$ collected 42 patients with UC who were ineffective in the treatment of mesalazine and belonged to the type of spleen kidney yang deficiency and damp heat accumulation. They were all given Yukui formula granules (Sanguisorba officinalis, Rhizoma Atractylodis Macrocephalae, Rhizoma Atractylodis Macrocephalae, Cortex Cinnamomi, Radix Glycyrrhizae, Rhizoma Zingiberis officinalis, Herba agrimoniae $20 \mathrm{~g}$, Rhizoma epiphyllae $6 \mathrm{~g}$, indigo naturalis $2 \mathrm{~g}$ ). After two months of treatment, it was concluded that Yukui formula had a good repair effect on the intestinal mucosal lesions of UC patients, It's main function is to reduce TLR4 and NF-K. the expression of B cells. Hu Zhifei ${ }^{[7]}$ and others selected 80 cases of UC of spleen kidney yang deficiency type. The control group was treated with sulfasalazine enteric coated tablets. The treatment group was treated with self-made Zhuyang Tongfu Decoction (Corydalis yanhusuo, cinnamon, fried Sophora japonica, dried ginger, cockscomb flower, Aucklandia, tangerine peel, fried Atractylodes macrocephala, Coptis, coix seed, fried Toona bark, Poria cocos 20g each, Amomum villosum, processed Pinellia ternata $15 \mathrm{~g}$ each, fried licorice $10 \mathrm{~g}$ each). After two weeks of treatment, the patients in the control group were treated with sulfasalazine enteric coated tablets, The total effective rate of the treatment group was $85.0 \%$, which was significantly higher than $72.5 \%$ of the control group, and the symptom score of the treatment group was better than that of the control group, which indicated that TCM could significantly improve the clinical symptoms of UC of spleen kidney yang deficiency type, which was worthy of popularization and application.

\subsection{External therapy external therapy of TCM is widely used in the treatment of UC with significant effect}

It mainly includes, acupoint application, TCM enema, acupuncture and moxibustion therapy, etc. Yan Chengqiu ${ }^{[8]}$ and others selected 36 patients with UC of spleen and kidney yang deficiency type. The control group was given mesalazine enteric coated tablets, and the treatment group was given TCM collapsing treatment (Eupatorium adenophorum, Artemisia argyi, Angelica dahurica, cardamom, dried ginger, Poria cocos, Evodia rutaecarpa, Gardenia jasminoides Ellis) on the basis of the control group. It was finally confirmed that TCM collapsing treatment had significant effect on UC of spleen and kidney yang deficiency type. Zhou Hua ${ }^{[9]}$ in the treatment of 30 cases of ulcerative colitis of spleen and kidney yang deficiency type with ginger and salt partitioned moxibustion, the treatment group selected Shuangtianshu, Shenque and Zhongwan for ginger and salt partitioned moxibustion for six weeks, and the control group took mesalazine orally. The results showed that the quantitative score of TCM symptom grading in the treatment group was lower than that in the control group after treatment. It can be seen that the external treatment of TCM is safe and effective in the treatment of UC, which is worthy of clinical application.

\section{Problems and prospects}

In conclusion, the treatment of UC by warming kidney and strengthening spleen should be further improved. The method, course of treatment and scheme of Wenshen Jianpi therapy are different, and most of them take the short-term clinical efficacy as the observation index, while the research on adverse reactions and disease recurrence rate is relatively less; The course of the disease is long, it is easy to relapse, the recurrence rate is high, and it needs long-term medication. Therefore, we should improve the level of clinical research 
on the premise of highlighting the characteristic treatment of warming kidney and strengthening spleen; In order to give full play to the advantages of TCM in the treatment of this disease, we should unify the standards and pay attention to the observation of long-term efficacy and adverse reactions.

\section{Disclosure statement}

The author declares no conflict of interest.

\section{References}

[1] Yang Q, Zhao Y, Bai Q, et al., 2018, Bian Songjing's Experience in Treating Ulcerative Colitis. Shanghai Journal of TCM, 52(11): 24-26.

[2] Xing W, Liu Y, 2019, A Clinical Case of Professor Liu Yanjing in Treating Ulcerative Colitis (Spleen Kidney Yang Deficiency Syndrome). Electronic Journal of Cardiovascular Disease of Integrated TCM and Western Medicine, 7(02): $159+161$.

[3] Xing W, 2019, Clinical Study on Treating Ulcerative Colitis (Spleen Kidney Yang Deficiency Syndrome) by Warming Kidney, Strengthening Spleen and Removing Dampness. Changchun University of TCM.

[4] He Qi, 2020, Clinical Study on Treating Ulcerative Colitis (Spleen Kidney Yang Deficiency Syndrome) with Renren yangzang Decoction and Nuangan Decoction. Changchun University of TCM.

[5] Lu M, 2019, Clinical Observation on the Treatment of Ulcerative Colitis (Spleen Kidney Yang Deficiency) with Sishen Pill. Changchun University of TCM.

[6] Zhang L, 2018 Effect of Yukui Recipe on Ulcerative Colitis with Ineffective Mesalazine and its Effect on TLR4 and NF in Intestinal Mucosa-K. Liaoning University of TCM.

[7] $\mathrm{Hu}$ Z, Zhang H, 2019, 40 Cases of Ulcerative Colitis of Spleen Kidney Yang Deficiency Type Treated with Zhuyang Tongfu Decoction. National Medical Forum, 34 (1): 27-28.

[8] Yan C, Bao H, Liu Y, et al., 2019, 36 Cases of Ulcerative Colitis of Yang Deficiency of Spleen and Kidney Treated with traditional Chinese medicine kuizi. Modern distance education of TCM, 17(5): 54-56.

[9] Zhou Hua, 2020, 30 Cases of Ulcerative Colitis of Yang Deficiency of Spleen and Kidney Treated with Ginger and Salt Partitioned Moxibustion. Massage and Rehabilitation Medicine, 11(23): 71$72+74$. 